\title{
ENDOCRINE SURGERY
}

Ann R Coll Surg Engl 2009; 91: 214-216

doi $10.1308 / 003588409 \times 391811$

\section{The use of the harmonic scalpel in thyroidectomy: 'beyond the learning' curve'}

\author{
E FOREMAN, S ASPINALL, RD BLISS, TWJ LENNARD
}

Department of Endocrine Surgery, Royal Victoria Infirmary, Newcastle upon Tyne, UK School of Surgical and Reproductive Sciences, University of Newcastle upon Tyne, Newcastle upon Tyne, UK

ABSTRACT

INTRODUCTION Safe and effective haemostasis in surgery is clearly essential, and in the neck where risks of airway compromise are also present any new technology that purports to offer advantages must be rigorously evaluated. We describe our experience with the use of the Harmonic Scalpel [Ethicon UK] in thyroidectomy.

PATIENTS AND METHODS A retrospective clinical review of 183 patients undergoing hemi or total thyroidectomies from 12 months prior to using the harmonic scalpel (2003; $n=77$ ) and 12 months 'beyond the learning curve' $(2006 ; n=106)$.

RESULTS The results demonstrate that, once past the learning curve, the use of the harmonic scalpel during thyroidectomy significantly reduces operative time and postoperative hypocalcaemia, and is as safe as conventional surgery with regard to voice change and bleeding.

CONCLUSIONS The harmonic scalpel is as safe as conventional methods of haemostasis and operations using this technique are quicker once the need to have repetitive 'clip, cut and tie' routines is avoided.

\section{KEYWORDS}

\section{Harmonic scalpel - Thyroidectomy - Haemostasis}

\section{CORRESPONDENCE TO}

TWJ Lennard, School of Surgical and Reproductive Sciences, Newcastle University of Newcastle upon Tyne, Newcastle upon Tyne NE2 4HH, UK. E: t.w.j.lennard@ncl.ac.uk

Traditional approaches to control of the vascular pedicles in thyroid surgery include ligation in continuity followed by division, 'clip, cut and ligate' sequences, and the use of small metallic clips to control vessels prior to division. These techniques are safe and effective with an overall reoperation rate for haemorrhage of $1.25 \%$ (British Association of Endocrine and Thyroid Surgeon's National Audit 2007). Effective haemostasis not only is a prerequisite of safe surgery but, in addition, aids the surgeon in identifying structures such as the parathyroid glands and recurrent laryngeal nerves. Meticulous attention to detail is paramount in thyroid surgery where a clear view of these structures is essential to prevent morbidity. In assessing any new method of haemostasis, therefore, it is important to evaluate not only the primary end-point of bleeding but also what might be called collateral damage to neighbouring tissues from, for example, heat, pressure or instrumentation.

Published experience suggests that the use of the harmonic scalpel (Ethicon UK) in a variety of surgical operations is safe and may reduce operative time. We report our results following 2 years' experience with the harmonic scalpel in thyroid surgery in a comparative study.

\section{Patients and Methods}

A retrospective clinical review of 183 patients undergoing hemi or total thyroidectomies from 12 months prior to using the harmonic scalpel (2003; $n=77)$ and 12 months 'beyond the learning curve' $(2006 ; n=106)$. In the two populations, we compared: (i) operative time (arrival in theatre to anaesthetic record end); (ii) postoperative serum ionised calcium at $24 \mathrm{~h}$ and $48 \mathrm{~h}$, the nadir being the value used irrespective of symptoms; (iii) re-operation rate for haemorrhage; and (iv) observed or reported voice change.

The main outcome measures were directly compared between the two cohorts using the two-tailed $t$-test. Statistical significance was assumed at $P<0.05$.

\section{Results}

All operations were performed or supervised by two experienced endocrine surgeons. The two patient cohorts were similar regarding age, sex and thyroid pathology. Re-operation for bleeding was one in the harmonic group and one in the pre-harmonic group. Reported voice change by the 


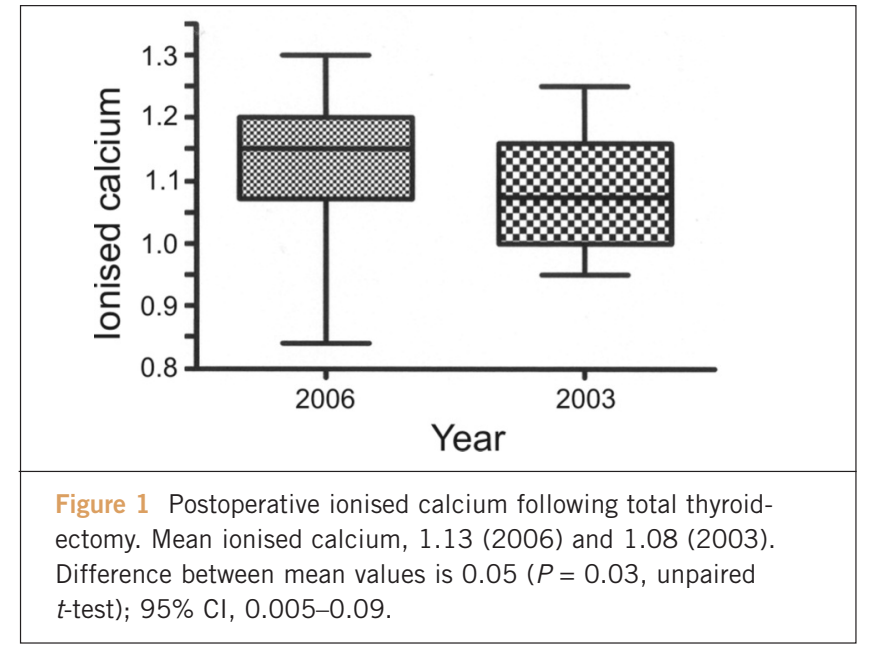

patient prompting investigation uncovered one transient, recurrent laryngeal nerve palsy in each group. There was reduced hypocalcaemia reflected in a significantly higher postoperative ionised calcium level following total thyroidectomy in the harmonic scalpel group $(P=0.03$, unpaired $t$-test) compared to the conventional pre-harmonic group (Fig. 1). The operations were quicker with the harmonic scalpel: the mean operating time of the harmonic scalpel group was 5 min less $(7.5 \%$ decrease; $P=0.2122$, unpaired $t$-test) in unilateral thyroid lobectomy (Fig. 2) and 11.29 min less $(12.0 \%$ decrease; $P=0.0036$, unpaired $t$-test) following total thyroidectomy (Fig. 3) when compared with the pre-harmonic group.

\section{Discussion}

The results demonstrate that, once past the learning curve, the use of the harmonic scalpel during thyroidectomy significantly reduces operative time and postoperative hypocalcaemia, and is as safe as conventional surgery with regard to voice change and bleeding.

The harmonic scalpel is commonly used in laparoscopic surgery. ${ }^{1}$ Its ultrasonically activated coagulation shears use high-frequency ultrasound ( $55 \mathrm{kHz}$ ) to divide vessels of up to $5 \mathrm{~mm}$ during thyroidectomy. The active blade vibrates longitudinally against an inactive blade, combining cutting and coagulation. It operates at a relatively low temperature $\left(80^{\circ} \mathrm{C}\right)$; consequently, there is potentially less thermal damage to surrounding tissues than electrocautery and laser. There are possible benefits of using the harmonic in thyroidectomy. First, a reduction in operative time, as repetitive 'clip, cut and tie' routines are avoided. ${ }^{2}$ This also permits less need for surgical assistants. The reduction in postoperative hypocalcaemia may occur as a consequence of less injury to the parathyroids and surrounding structures through lateral dispersion of heat. Excellence in haemostasis may also permit a better view of these and other impor-
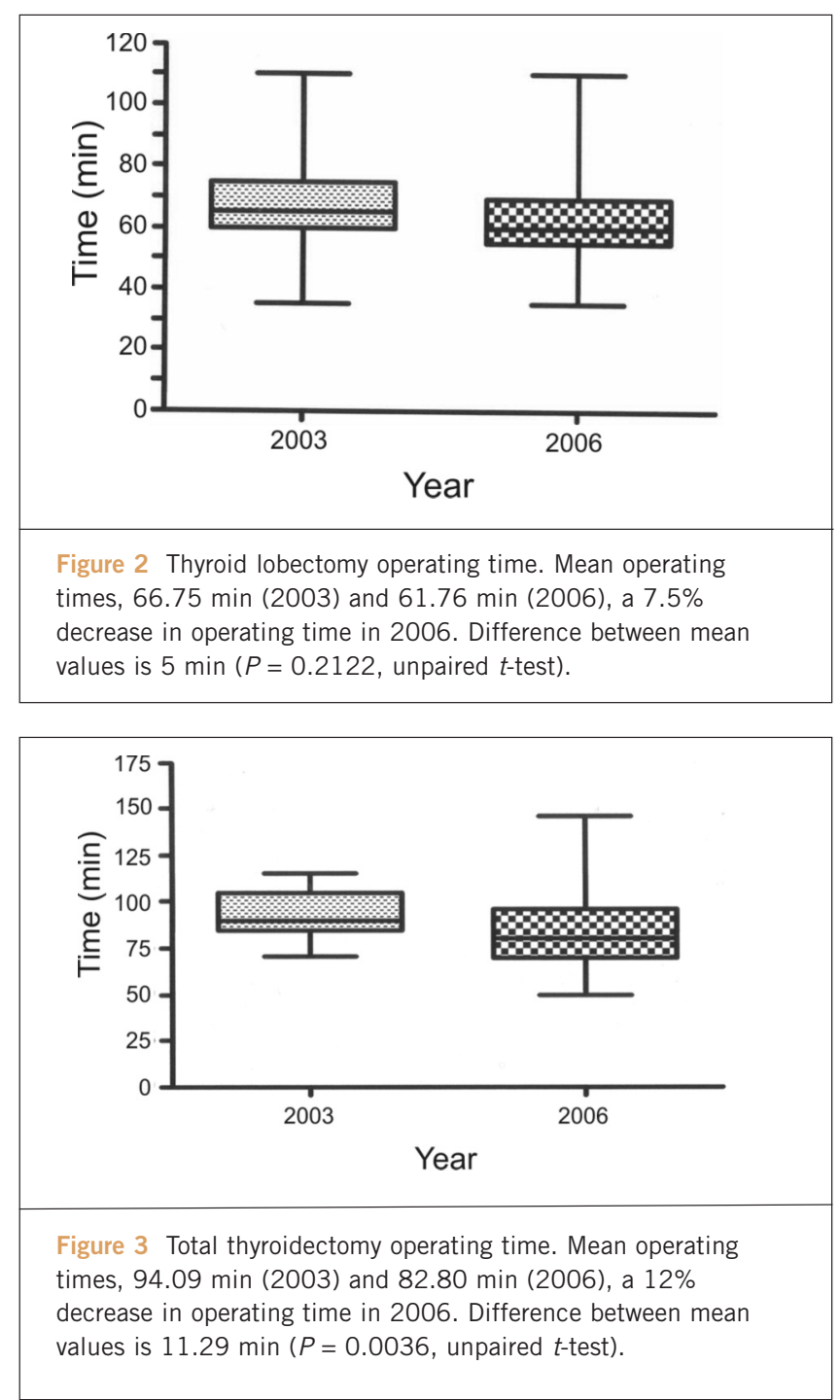

tant structures to preserve when operating. However, potential increased cost and the skill required in overcoming the learning curve are possible disadvantages. Although no definition of the learning curve for this technique exists (and is likely to be different for each surgeon), we report our results here 'beyond the learning curve' following 2 years' experience with the harmonic scalpel during thyroid surgery, by which time the technique was established in our unit and in routine use.

The difficulties of performing a randomised, controlled trial in comparing surgical techniques are self-evident. The outcomes of a novel technique might be confounded by the learning curve phenomenon. ${ }^{3}$ Once the novel technique is established, advantages may be seen and randomisation at this point becomes difficult. We undertook a retrospective review of patients undergoing thyroid surgery over two separate 12-month periods. The first cohort was taken from 2003 and consisted of 77 patients. This group underwent 
surgery prior to the introduction of the harmonic scalpel, using conventional 'clip, cut and tie' routines with additional small metal clips (Ligaclips, Ethicon UK) to secure haemostasis. The second cohort included 106 patients whose surgery was performed in 2006, two years following experience of the harmonic scalpel, at a time when all involved felt comfortable with the technology.

With regard to cost, the contract price for the disposable items and generator will vary across different health authorities, but constant in the equation will be the absence of metal clips, sutures or ties, a quicker turnaround time permitting on average another case to be done per list, ${ }^{4}$ and less hospital stay and expense relating to the correction of hypocalcaemia.

\section{Conclusions}

The harmonic scalpel is as safe as conventional methods of haemostasis, is associated with less postoperative hypocalcaemia and operations using this technique are quicker once the need to have repetitive 'clip, cut and tie' routines is avoided.

\section{Acknowledgement}

TWJL teaches at the European Surgical Institute, Hamburg, for which he receives an honorarium from Ethicon UK.

\section{References}

1. Valeri A, Borelli A, Presenti L, Lucchese M, Manca G, Tonelli P et al. The influence of new technologies on laparoscopic adrenalectomy. Our personal experience with 91 patients. Surg Endosc 2002; 16: 1274-9.

2. Voutilainen PE, Haapianen R, Haglund C. Ultrasonically activated shears in thyroid surgery. Am J Surg 1998; 175: 491-3.

3. Cordon C, Fajardo R, Ramirex J, Herrera MF. A randomized, prospective, parallel group study comparing the harmonic scalpel to electrocautery in thyroidectomy. Surgery 2005; 137: 337-41.

4. Shemen L. Thyroidectomy using the harmonic scalpel: analysis of 105 consecutive cases. Otolaryngol Head Neck Surg 2002; 127: 284-8.

\section{New online only case reports}

You can access the case reports by using your College-issued Athens username and password to enter the members' area of the College website (<www.rcseng.ac.uk/members/annals/>) and following the link to the Annals.

Alternatively, if you type the following URL into the address bar of your web browser <http://dx.doi.org/> and then enter the DOI in the dialogue box presented on this web page, you will be taken directly to the abstract of the article. 